\title{
Some Remarks on Functionals with the Tensorization Property
}

by

\author{
Paweł WOLFF \\ Presented by Stanisław KWAPIEŃ
}

\begin{abstract}
Summary. We investigate the subadditivity property (also known as the tensorization property) of $\varphi$-entropy functionals and their iterations. In particular we show that the only iterated $\varphi$-entropies with the tensorization property are iterated variances. This is a complement to the result due to Latała and Oleszkiewicz on characterization of the standard $\varphi$-entropies with the tensorization property.
\end{abstract}

1. Introduction. An important feature of some functional inequalities for probability measures is the tensorization property (sometimes called the product property): if the inequality holds for each measure $\mu_{1}, \mu_{2}, \ldots$ then it also holds for the product measure $\mu_{1} \otimes \mu_{2} \otimes \cdots$. In this paper we focus on the tensorization property of entropy-energy inequalities, well-known examples of which are the logarithmic Sobolev inequality and Poincare inequality.

By the $\varphi$-entropy functional we mean the functional $E \varphi(Z)-\varphi(E Z)$. For $\varphi(x)=x \log x$ we get the classical entropy functional, for $\varphi(x)=x^{2}$ we get the variance, and for $\varphi(x)=x^{p}, p \in(1,2]$, the so-called $p$-variance. The family of entropy-energy inequalities corresponding to the $p$-variance, which interpolate between the logarithmic Sobolev and Poincaré inequalities, was introduced by Beckner [1] in the context of Gaussian measure on $\mathbb{R}^{n}$ and Haar measure on the sphere $S^{n-1}$. A more abstract treatment of this family of inequalities (in the context of arbitrary probability measures) was given by Latała and Oleszkiewicz [3]. One of the results in that paper states that if $\varphi:(0, \infty) \rightarrow \mathbb{R}$ belongs to the class $\Phi$, that is, $\varphi$ is either affine or convex with

2000 Mathematics Subject Classification: Primary 60E15; Secondary 28A35, 52A40.

Key words and phrases: product measure, convex functional, entropy, non-negativity, tensorization property.

Research partially supported by MEiN Grant 1 PO3A 01229. 
$1 / \varphi^{\prime \prime}$ concave, then the $\varphi$-entropy functional has the tensorization property, i.e. for any random variable $Z$ defined on any product space $\Omega_{1} \times \Omega_{2}$,

$$
E \varphi(Z)-\varphi(E Z) \leq E\left[\left(E_{1} \varphi(Z)-\varphi\left(E_{1} Z\right)\right)+\left(E_{2} \varphi(Z)-\varphi\left(E_{2} Z\right)\right)\right],
$$

or, equivalently,

$$
\Psi_{2}(Z)=E \varphi(Z)-E_{1} \varphi\left(E_{2} Z\right)-E_{2} \varphi\left(E_{1} Z\right)+\varphi(E Z) \geq 0 .
$$

(The solution of a similar characterization problem, concerning hypercontractivity with some more general functionals instead of $L_{p}$ norms, was given by Oleszkiewicz [6]). In fact, the paper [3] contains a rigorous proof only of the statement that if $\varphi \in \Phi$ then the $\varphi$-entropy functional

$$
\Psi_{1}(Z)=E \varphi(Z)-\varphi(E Z) \text { is convex. }
$$

Later on, in [2] it was suggested that the convexity of $\Psi_{1}$ might not imply the non-negativity of $\Psi_{2}$ straightforwardly. Therefore in order to obtain the latter, a variational formula for $\Psi_{2}$ was used (established by Bobkov for some particular functions $\varphi$; see [4, Section 4]). However, this formula strongly relies on the analytic conditions that $\varphi$ satisfies (namely, that $\varphi \in \Phi$ ).

In order to make the picture clear, we shall provide a direct argument that the convexity of $\Psi_{1}$ is equivalent to the non-negativity of $\Psi_{2}$ (Proposition 1). We also give the proof of the converse part of the characterization result (Theorem 1): if the $\varphi$-entropy has the tensorization property (in other words, $\varphi$ belongs to the class $C_{2}$ ) then $\varphi \in \Phi$. Finally, Theorem 2 addresses the question posed at the end of [3], concerning a characterization of the higher "tensorization classes" $C_{n}$ for $n>2$.

2. Notation and definitions. Throughout the paper, $d$ and $n$ stand for positive integers, $U$ denotes an open, convex subset of $\mathbb{R}^{d}$ and $\varphi: U \rightarrow \mathbb{R}$ is a continuous function. By $(\Omega, \mathcal{F}, P),\left(\Omega_{k}, \mathcal{F}_{k}, P_{k}\right)$, etc. we shall denote probability spaces. In the case of the product space $(\Omega, \mathcal{F}, P)=\bigotimes_{k=1}^{n}\left(\Omega_{k}, \mathcal{F}_{k}, P_{k}\right)$, for $K \subset\{1, \ldots, n\}, E_{K}$ stands for the expectation with respect to the product measure $\bigotimes_{k \in K} P_{k}$. For $k \in\{1, \ldots, n\}$ we shall write $E_{k}$ instead of $E_{\{k\}}$.

For $V \subseteq \mathbb{R}^{d}$, when writing $Z:(\Omega, \mathcal{F}, P) \rightarrow V$, we mean that $Z$ is a random variable taking values in $\mathbb{R}^{d}$ and $P(Z \in V)=1$.

For fixed $U \subseteq \mathbb{R}^{d}, \varphi: U \rightarrow \mathbb{R}$ and fixed $(\Omega, \mathcal{F}, P)=\bigotimes_{k=1}^{n}\left(\Omega_{k}, \mathcal{F}_{k}, P_{k}\right)$ we shall consider the functional $\Psi_{n}$ acting on random variables $Z$ defined on $(\Omega, \mathcal{F}, P)$ with $P(Z \in V)=1$ for some compact, convex set $V \subset U$, and defined by

$$
\Psi_{n}(Z)=\sum_{K \subseteq\{1, \ldots, n\}}(-1)^{|K|} E_{K^{c}} \varphi\left(E_{K} Z\right)
$$

The definition of the main object we investigate in this paper originates in [3]: 
Definition 1. We say that $\varphi \in C_{n}(U)$ iff the functional $\Psi_{n}$ is nonnegative for any $(\Omega, \mathcal{F}, P)=\bigotimes_{k=1}^{n}\left(\Omega_{k}, \mathcal{F}_{k}, P_{k}\right)$, i.e. for every compact, convex set $V \subset U$ and every $Z:(\Omega, \mathcal{F}, P) \rightarrow V$,

$$
\Psi_{n}(Z) \geq 0 .
$$

REMARK 1. It is obvious that $C_{n}(U)$ is a convex cone.

REMARK 2. By slight abuse of notation, we can also define the functional $\Psi_{n}$ inductively, as iterations of the $\varphi$-entropy functional $E \varphi(Z)-\varphi(E Z)$, namely

$$
\Psi_{n}(Z)=E_{n} \Psi_{n-1}(Z)-\Psi_{n-1}\left(E_{n} Z\right) .
$$

(By $\Psi_{n-1}(Z)$ we mean the application of $\Psi_{n-1}$ conditionally with the $n$th product coordinate fixed, whereas in $\Psi_{n-1}\left(E_{n} Z\right)$ we consider $E_{n} Z$ as a random variable defined on the product of all probability spaces except the $n$ th). Now, it can be seen that the non-negativity of $\Psi_{n}$ is tightly connected with the convexity of $\Psi_{n-1}$. A precise statement appears in Proposition 1 (equivalence of (i) and $\left(\mathrm{ii}^{\prime}\right)$ ).

REMARK 3 . The functional $\Psi_{n}$ can be extended to a functional $\widetilde{\Psi}_{n}$ acting on a larger class of random variables whose values are not restricted almost surely to some compact subset of $U$. However, some integrability assumptions should be added to ensure that the right hand side of (1) is well-defined. It would be natural to assume that $\varphi$ is convex, $E|Z|<\infty(|\cdot|$ stands for Euclidean norm in $\mathbb{R}^{d}$ ) and $E|\varphi(Z)|<\infty$. Then Jensen's inequality implies that for each $K \subseteq\{1, \ldots, n\}$,

$$
a E_{K} Z+b \leq \varphi\left(E_{K} Z\right) \leq E_{K} \varphi(Z) \quad \text { a.s. }
$$

for some $a, b \in \mathbb{R}$. Since the lower and upper bounds are integrable with respect to $E_{K^{c}}$, each term in the sum (1) is well-defined and finite. As we shall see, in the context of the classes $C_{n}(U)$, the assumption that $\varphi$ is convex is not restrictive at all. Moreover, an easy truncation argument will show that the non-negativity of $\widetilde{\Psi}_{n}$ is a consequence of the non-negativity of $\Psi_{n}$ (see Proposition 1, equivalence of (i) and (iii)).

EXAmPle 1. Jensen's inequality implies that $C_{1}(U)$ contains exactly the convex functions on $U$.

EXAmple 2. The class $C_{2}((0, \infty))$ is exactly the class of functions $\varphi$ for which the subadditive $\varphi$-entropies are widely considered. The most important examples are $\varphi(x)=x^{p}$ for $p \in(1,2]$ and $\varphi(x)=x \log (x)$. In the introduction we mentioned that $\Phi \subseteq C_{2}((0, \infty))$. In fact, we shall show that these two classes are equal (see Theorem 1 ). 
3. Properties of the classes $C_{n}$. We start with a proposition giving some equivalent variants of the definition of the class $C_{n}$. The discrete cubes $\{-1,1\}_{\lambda}^{n}$ considered below are the $n$-fold products of the two-point probability space $\{-1,1\}$ endowed with the measure $\lambda \delta_{1}+(1-\lambda) \delta_{-1}$; if $\lambda$ is omitted then it means that we take $\lambda=1 / 2$.

Proposition 1. The following assertions are equivalent:

(i) $\varphi \in C_{n}(U)$,

(ii) for every random variable $Z:\{-1,1\}^{n} \rightarrow U$ we have $\Psi_{n}(Z) \geq 0$,

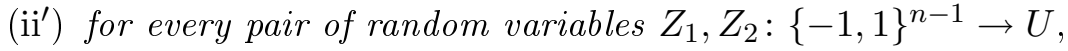

$$
\frac{1}{2} \Psi_{n-1}\left(Z_{1}\right)+\frac{1}{2} \Psi_{n-1}\left(Z_{2}\right) \geq \Psi_{n-1}\left(\frac{Z_{1}+Z_{2}}{2}\right),
$$

(iii) $\varphi$ is convex and for every $(\Omega, \mathcal{F}, P)=\bigotimes_{k=1}^{n}\left(\Omega_{k}, \mathcal{F}_{k}, P_{k}\right)$ and every random variable $Z:(\Omega, \mathcal{F}, P) \rightarrow U$ such that $E|Z|<\infty$ and $E|\varphi(Z)|<\infty$ we have $\widetilde{\Psi}_{n}(Z) \geq 0$.

In the proof we shall use the following lemmas:

Lemma 1. Let $V$ be a compact, convex subset of $\mathbb{R}^{d}$ and $(\Omega, \mathcal{F}, P)$ $=\left(\Omega_{1}, \mathcal{F}_{1}, P_{1}\right) \otimes\left(\Omega_{2}, \mathcal{F}_{2}, P_{2}\right)$ be a product probability space. For every $Z$ : $(\Omega, \mathcal{F}, P) \rightarrow V$ and every $\varepsilon>0$ there exists $\widetilde{Z}:(\Omega, \mathcal{F}, P) \rightarrow V$ such that

$$
\widetilde{Z}=\sum_{i=1}^{M} \sum_{j=1}^{N} a_{i j} 1_{A_{i} \times B_{j}},
$$

where $a_{i j} \in V$ and $\left(A_{i}\right)_{i=1}^{M},\left(B_{j}\right)_{j=1}^{N}$ are measurable, finite partitions of $\left(\Omega_{1}, \mathcal{F}_{1}, P_{1}\right)$ and $\left(\Omega_{2}, \mathcal{F}_{2}, P_{2}\right)$ (respectively), and $P(|\widetilde{Z}-Z| \geq \varepsilon)<\varepsilon$.

Proof. We take any $\varepsilon>0$ and any finite covering of $V$ by (open) balls $U_{i}=B\left(a_{i}, \varepsilon\right)(i=1, \ldots, L)$ such that $a_{i} \in V$. Then we take disjoint and measurable (with respect to $\left.\mathcal{F}_{1} \otimes \mathcal{F}_{2}\right)$ sets $C_{i}=Z^{-1}\left(U_{i} \backslash \bigcup_{j<i} U_{j}\right)$. Now we shall represent each $C_{i}$ as a union of finitely many measurable product sets $A \times B$ in such a way that the measure of the symmetric difference of this union and $C_{i}$ is small. Since $P_{1} \otimes P_{2}$ is the product measure, we can find countably many sets $A_{i, j} \in \mathcal{F}_{1}$ and $B_{i, j} \in \mathcal{F}_{2}(j=1,2, \ldots)$ such that $C_{i} \subseteq \bigcup_{j=1}^{\infty}\left(A_{i, j} \times B_{i, j}\right)$ and

$$
P\left(C_{i}\right)+\varepsilon / L^{2}>\sum_{j=1}^{\infty} P_{1}\left(A_{i, j}\right) P_{2}\left(B_{i, j}\right) .
$$

If we take $m_{i}$ such that the tail of the above series for $j>m_{i}$ is less than $\varepsilon / L^{2}$ and put $\widetilde{C}_{i}=\bigcup_{j=1}^{m_{i}}\left(A_{i, j} \times B_{i, j}\right)$, then 


$$
\begin{aligned}
& P\left(C_{i} \backslash \widetilde{C}_{i}\right) \leq P\left(\bigcup_{j>m_{i}}\left(A_{i, j} \times B_{i, j}\right)\right)<\varepsilon / L^{2}, \\
& P\left(\widetilde{C}_{i} \backslash C_{i}\right) \leq P\left(\bigcup_{j=1}^{\infty}\left(A_{i, j} \times B_{i, j}\right)\right)-P\left(C_{i}\right)<\varepsilon / L^{2} .
\end{aligned}
$$

We set

$$
D_{i}=\widetilde{C}_{i} \backslash \bigcup_{i^{\prime} \neq i} \widetilde{C}_{i^{\prime}} \quad \text { for } i=1, \ldots, L .
$$

Obviously, the $D_{i}$ are pairwise disjoint and each of them is a finite union of measurable product sets. Putting $D_{0}=\Omega \backslash \sum_{i=1}^{L} D_{i}$ (which is also a finite union of product sets) and choosing an arbitrary $a_{0} \in V$, we see that $\widetilde{Z}=$ $\sum_{i=0}^{L} a_{i} 1_{D_{i}}$ has the desired form (to see this, take a joint subdivision of $\Omega_{1}$ and $\Omega_{2}$ generated by all (finitely many) product sets from $D_{0}, D_{1}, \ldots, D_{L}$ ).

To finish the proof we show that $P(|\widetilde{Z}-Z| \geq \varepsilon)<\varepsilon$. For each $i$ we have

$$
\begin{aligned}
\{|\widetilde{Z}-Z| \geq \varepsilon\} \cap C_{i} & \subseteq C_{i} \backslash D_{i}=\left(C_{i} \backslash \widetilde{C}_{i}\right) \cup \bigcup_{i^{\prime} \neq i}\left(C_{i} \cap \widetilde{C}_{i^{\prime}}\right) \\
& \subseteq\left(C_{i} \backslash \widetilde{C}_{i}\right) \cup \bigcup_{i^{\prime} \neq i}\left(\widetilde{C}_{i^{\prime}} \backslash C_{i^{\prime}}\right),
\end{aligned}
$$

since $C_{i} \cap \widetilde{C}_{i^{\prime}}=\left(\widetilde{C}_{i^{\prime}} \backslash C_{i^{\prime}}\right) \cap C_{i} \subseteq \widetilde{C}_{i^{\prime}} \backslash C_{i^{\prime}}$. Therefore for each $i=1, \ldots, L$, (3) and (4) yield

$$
P\left(\{|\widetilde{Z}-Z| \geq \varepsilon\} \cap C_{i}\right) \leq P\left(C_{i} \backslash \widetilde{C}_{i}\right)+\sum_{i^{\prime} \neq i} P\left(\widetilde{C}_{i^{\prime}} \backslash C_{i^{\prime}}\right)<\varepsilon / L .
$$

Lemma 2. Let $V$ be a compact, convex subset of $\mathbb{R}^{d}$ and $\varphi: V \rightarrow \mathbb{R}$ be a continuous function. If the sequence $Z_{k}:\left(\Omega_{1}, \mathcal{F}_{1}, P_{1}\right) \otimes\left(\Omega_{2}, \mathcal{F}_{2}, P_{2}\right) \rightarrow V$ converges in probability to $Z$ then $E_{1} \varphi\left(E_{2} Z_{k}\right) \rightarrow E_{1} \varphi\left(E_{2} Z\right)$.

Proof. Let $R>0$ satisfy $V \subseteq B(0, R)$. We take any $\varepsilon>0$ and $k$ such that $P\left(\left|Z_{k}-Z\right| \geq \varepsilon\right)<\varepsilon$. Consider the measurable sets $A=\left\{\left|Z_{k}-Z\right| \geq \varepsilon\right\}$ $\subseteq \Omega_{1} \times \Omega_{2}$ and $A_{\omega_{1}}=\left\{\omega_{2}:\left(\omega_{1}, \omega_{2}\right) \in A\right\} \subseteq \Omega_{2}$ for each $\omega_{1} \in \Omega_{1}$. By Fubini's theorem we get

$$
\varepsilon>E 1_{A}=\int_{\Omega_{1}} P_{2}\left(A_{\omega_{1}}\right) P_{1}\left(d \omega_{1}\right) \geq \sqrt{\varepsilon} P_{1}(B),
$$

where $B=\left\{\omega_{1}: P_{2}\left(A_{\omega_{1}}\right) \geq \sqrt{\varepsilon}\right\}$ is a measurable subset of $\Omega_{1}$, which yields $P_{1}(B)<\sqrt{\varepsilon}$. Now we write

$$
\begin{aligned}
\left|E_{1} \varphi\left(E_{2} Z_{k}\right)-E_{1} \varphi\left(E_{2} Z\right)\right| \leq & \int_{B} \mid \varphi\left(E_{2} Z_{k}\left(\omega_{1}, \cdot\right)-\varphi\left(E_{2} Z\left(\omega_{1}, \cdot\right) \mid P_{1}\left(d \omega_{1}\right)\right.\right. \\
& +\int_{\Omega_{1} \backslash B} \mid \varphi\left(E_{2} Z_{k}\left(\omega_{1}, \cdot\right)-\varphi\left(E_{2} Z\left(\omega_{1}, \cdot\right) \mid P_{1}\left(d \omega_{1}\right),\right.\right.
\end{aligned}
$$


and the first term on the right hand side can be estimated by $2 P_{1}(B) \sup _{V}|\varphi|$ $<2 \sqrt{\varepsilon} \sup _{V}|\varphi|$. For $\omega_{1} \notin B$,

$$
\begin{aligned}
\left|E_{2} Z_{k}\left(\omega_{1}, \cdot\right)-E_{2} Z\left(\omega_{1}, \cdot\right)\right| & \leq E_{2}\left(\left\|Z_{k}\right\|_{\infty}+\|Z\|_{\infty}\right) 1_{A_{\omega_{1}}}+\varepsilon E_{2} 1_{\Omega_{2} \backslash A_{\omega_{1}}} \\
& <2 R \sqrt{\varepsilon}+\varepsilon,
\end{aligned}
$$

and the uniform continuity of $f$ yields

$$
\left|E_{1} \varphi\left(E_{2} Z_{k}\right)-E_{1} \varphi\left(E_{2} Z\right)\right|<2 \sqrt{\varepsilon} \sup _{V}|\varphi|+\delta(2 R \sqrt{\varepsilon}+\varepsilon) \rightarrow 0 \quad \text { as } \varepsilon \rightarrow 0,
$$

where $\delta(\varepsilon)$ is the modulus of continuity of $\varphi$.

Proof of Proposition 1. The implications (i) $\Rightarrow$ (ii), (iii) $\Rightarrow$ (i), (iii) $\Rightarrow$ (ii) and (ii) $\Leftrightarrow\left(\mathrm{ii}^{\prime}\right)$ are obvious. The proof of the implication (i) $\Rightarrow$ (iii) is postponed until after the proof of Proposition 2. Now we prove (ii) $\Rightarrow$ (i). It suffices to show that for any fixed compact, convex $V \subset U$ and any fixed $\left(\Omega_{k}, \mathcal{F}_{k}, P_{k}\right)$ (for $k=1, \ldots, n-1$ ),

$$
\begin{aligned}
& \Psi_{n}(Z) \geq 0 \text { for every } Z: \bigotimes_{k=1}^{n-1}\left(\Omega_{k}, \mathcal{F}_{k}, P_{k}\right) \otimes\{-1,1\} \rightarrow V \\
& \quad \Rightarrow \Psi_{n}(Z) \geq 0 \text { for every }\left(\Omega_{n}, \mathcal{F}_{n}, P_{n}\right) \text { and } Z: \bigotimes_{k=1}^{n}\left(\Omega_{k}, \mathcal{F}_{k}, P_{k}\right) \rightarrow V
\end{aligned}
$$

which means that the convexity of $\Psi_{n-1}$ (even just $1 / 2$-convexity) implies the non-negativity of $\Psi_{n}$. Applying this argument $n$ times we get (i).

First note that the implication (5) holds for $\left(\Omega_{n}, \mathcal{F}_{n}, P_{n}\right)=\{-1,1\}_{\lambda}$ with $\lambda \in(0,1)$. Indeed, the hypothesis of (5) states that for any pair of random variables $Z_{1}, Z_{2}: \bigotimes_{k=1}^{n-1}\left(\Omega_{k}, \mathcal{F}_{k}, P_{k}\right) \rightarrow V$,

$$
\lambda \Psi_{n-1}\left(Z_{1}\right)+(1-\lambda) \Psi_{n-1}\left(Z_{2}\right) \geq \Psi_{n-1}\left(\lambda Z_{1}+(1-\lambda) Z_{2}\right)
$$

for $\lambda=1 / 2$, hence also for any $\lambda=j_{i} 2^{-i}\left(0<j_{i}<2^{i}\right)$. Letting $\lambda_{i} \rightarrow \lambda$ we get (6) for any $\lambda \in[0,1]$, because $X_{i}:=\lambda_{i} Z_{1}+\left(1-\lambda_{i}\right) Z_{2} \rightarrow \lambda Z_{1}+(1-\lambda) Z_{2}$ $=: X$ a.s., so $E_{K} X_{i} \rightarrow E_{K} X$ a.s. (the sequence $\left(X_{i}\right)$ is bounded a.s.) and also $E_{K^{\mathrm{c}}} \varphi\left(E_{K} X_{i}\right) \rightarrow E_{K^{\mathrm{c}}} \varphi\left(E_{K} X\right)$ ( $\varphi$ is continuous and bounded on $V$ ).

Now we show that $\left(\Omega_{n}, \mathcal{F}_{n}, P_{n}\right)$ can be an arbitrary probability space. Fix any $Z: \bigotimes_{k=1}^{n}\left(\Omega_{k}, \mathcal{F}_{k}, P_{k}\right) \rightarrow V$. Lemma 1 implies that for any $\varepsilon>0$ we may take $\widetilde{Z}: \bigotimes_{k=1}^{n}\left(\Omega_{k}, \mathcal{F}_{k}, P_{k}\right) \rightarrow V$ such that $P(|\widetilde{Z}-Z| \geq \varepsilon)<\varepsilon$ and

$$
\widetilde{Z}\left(\omega^{\prime}, \omega_{n}\right)=\sum_{j=1}^{N} \widetilde{Z}_{j}\left(\omega^{\prime}\right) 1_{B_{j}}\left(\omega_{n}\right),
$$

where $\widetilde{Z}_{j}: \bigotimes_{k=1}^{n-1}\left(\Omega_{k}, \mathcal{F}_{k}, P_{k}\right) \rightarrow V, \omega^{\prime} \in \prod_{k=1}^{n-1} \Omega_{k},\left(B_{j}\right)_{j=1}^{N}$ is a finite, measurable partition of $\left(\Omega_{n}, \mathcal{F}_{n}, P_{n}\right)$, and $\omega_{n} \in \Omega_{n}$. Then applying (6) $N-1$ 
times we get

$$
\begin{aligned}
E_{n} \Psi_{n-1}(\widetilde{Z}) & =\sum_{j=1}^{N} P_{n}\left(B_{j}\right) \Psi_{n-1}\left(\widetilde{Z}_{j}\right) \geq \Psi_{n-1}\left(\sum_{j=1}^{N} P_{n}\left(B_{j}\right) \Psi_{n-1}\left(\widetilde{Z}_{j}\right)\right) \\
& =\Psi_{n-1}\left(E_{n} \widetilde{Z}\right)
\end{aligned}
$$

hence, due to $(2), \Psi_{n}(\widetilde{Z}) \geq 0$. Lemma 2 implies that $\mid E_{K^{\mathrm{c}}} \varphi\left(E_{K} \widetilde{Z}\right)-$ $E_{K^{\mathrm{c}}} \varphi\left(E_{K} Z\right) \mid$ is small for each $K \subseteq\{1, \ldots, n\}$, hence letting $\varepsilon \rightarrow 0$ we obtain $\Psi_{n}(Z) \geq 0$.

Proposition 2. $C_{n+1}(U) \subseteq C_{n}(U)$.

Proof. Let $\varphi \in C_{n+1}(U)$. By Proposition 1 it is sufficient to show that $\Psi_{n}(Z) \geq 0$ for any $Z$ defined on $\Omega=\{-1,1\}^{n}$ taking values in $U$. Define $\bar{Z}$ on the $(n+1)$-fold product $\{-1,1\}^{n} \times \Omega$ by

$$
\bar{Z}\left(\varepsilon_{1}, \ldots, \varepsilon_{n}, \bar{\varepsilon}\right)=Z\left(\varepsilon_{1} \bar{\varepsilon}_{1}, \ldots, \varepsilon_{n} \bar{\varepsilon}_{n}\right),
$$

where $\varepsilon_{k} \in\{-1,1\}$ and $\bar{\varepsilon}=\left(\bar{\varepsilon}_{1}, \ldots, \bar{\varepsilon}_{n}\right) \in \Omega$. Since $\varphi \in C_{n+1}(U)$, we have $\Psi_{n+1}(\bar{Z})=E_{n+1} \Psi_{n}(\bar{Z})-\Psi_{n}\left(E_{n+1} \bar{Z}\right) \geq 0$. But $\Psi_{n}(\bar{Z}(\cdot, \bar{\varepsilon}))$ does not depend on the choice of $\bar{\varepsilon}$ and is equal to $\Psi_{n}(Z)$. Similarly $E_{n+1} \bar{Z}\left(\varepsilon_{1}, \ldots, \varepsilon_{n}, \cdot\right)$ does not depend on $\varepsilon_{k}$ and is equal to $E Z$, so we obtain $\Psi_{n+1}(\bar{Z})=\Psi_{n}(Z)$.

Now we can finish the proof of Proposition 1 :

Proof of Proposition 1, $(i) \Rightarrow\left(\right.$ iii). Fix any $\varphi \in C_{n}(U),(\Omega, \mathcal{F}, P)=$ $\bigotimes_{k=1}^{n}\left(\Omega_{k}, \mathcal{F}_{k}, P_{k}\right)$ and $Z:(\Omega, \mathcal{F}, P) \rightarrow U$ such that $E|Z|<\infty$ and $E|\varphi(Z)|<\infty$. Proposition 2 implies that $\varphi \in C_{1}(U)$, i.e. $\varphi$ is convex. Take any increasing sequence of compact, convex subsets $V_{i} \subset U$ such that $\bigcup_{i} V_{i}=U$, and fix $v_{0} \in V_{1}$. Then we define

$$
Z_{i}=Z 1_{Z \in V_{i}}+v_{0} 1_{Z \notin V_{i}}
$$

which converges to $Z$ a.s. We shall prove that

$$
E_{K^{\mathrm{c}}} \varphi\left(E_{K} Z_{i}\right) \rightarrow E_{K^{\mathrm{c}}} \varphi\left(E_{K} Z\right)
$$

which obviously implies that $\Psi_{n}\left(Z_{i}\right) \rightarrow \widetilde{\Psi}_{n}(Z)$. Since $\left|Z_{i}\right| \leq|Z|+\left|v_{0}\right|$ and $E_{K}|Z|<\infty$ a.s., Lebesgue's dominated convergence theorem implies that $E_{K} Z_{i} \rightarrow E_{K} Z$ a.s. and by continuity of $\varphi$ also $\varphi\left(E_{K} Z_{i}\right) \rightarrow \varphi\left(E_{K} Z\right)$ a.s. The convexity of $\varphi$ yields

$$
a E_{K} Z_{i}+b \leq \varphi\left(E_{K} Z_{i}\right) \leq E_{K} \varphi\left(Z_{i}\right)
$$

for some $a, b \in \mathbb{R}$. Since $E_{K} \varphi\left(Z_{i}\right) \leq E_{K}|\varphi(Z)|+\varphi\left(v_{0}\right)$ and $\left|a E_{K} Z_{i}+b\right|$ $\leq|a|\left(E_{K}|Z|+\left|v_{0}\right|\right)+|b|$ and both upper bounds are integrable with respect to $E_{K^{\mathrm{c}}}$, Lebesgue's theorem applied once again gives $E_{K^{\mathrm{c}}} \varphi\left(E_{K} Z_{i}\right) \rightarrow$ $E_{K^{\mathrm{c}}} \varphi\left(E_{K} Z\right)$.

From now on, we shall write $\Psi_{n}$, even if we really mean the extension $\widetilde{\Psi}_{n}$. 
We should mention that e.g. in the case of the class $C_{2}((0, \infty))$ one may have $\Psi_{2}(Z) \geq 0$ not only for $Z>0$ a.s., but also for $Z$ having an atom at 0 , as long as $\varphi$ can be extended continuously to $[0, \infty)$ (cf. Example 2). Generally, we can state the following

REMARK 4. If $\varphi: U \rightarrow \mathbb{R}$ extends continuously to $\bar{\varphi}: \bar{U} \rightarrow \mathbb{R}$, then $\varphi \in C_{n}(U)$ implies that $\Psi_{n}(Z) \geq 0$ for every random variable $Z$ defined on an $n$-fold product space and taking values in $\bar{U}$ and satisfying $E|Z|<\infty$ and $E|\bar{\varphi}(Z)|<\infty$. (More precisely, $\Psi_{n}$ here is a natural extension of the functional (1).) Indeed, since $\varphi \in C_{1}(U), \bar{\varphi}$ is also convex. Fixing $v_{0} \in U$ and defining $Z_{\varepsilon}=Z 1_{\{Z \notin \partial U\}}+\left((1-\varepsilon) Z+\varepsilon v_{0}\right) 1_{\{Z \in \partial U\}}$ for $\varepsilon \in(0,1)$ we obtain random variables $Z_{\varepsilon}$ with values in $U$ converging to $Z$ a.s. The proof that $\Psi_{n}\left(Z_{\varepsilon}\right) \rightarrow \Psi_{n}(Z)$ as $\varepsilon \rightarrow 0$ is the same as in the case of (7).

THEOREM 1. Let $U=(a, b) \subseteq \mathbb{R}$ be an open interval (possibly with $a=-\infty$ or $b=\infty)$ and let $\varphi: U \rightarrow \mathbb{R}$ be a continuous function. Then $\varphi \in C_{2}(U)$ iff $\varphi$ is an affine function or $\varphi$ is twice differentiable with $\varphi^{\prime \prime}>0$ and $1 / \varphi^{\prime \prime}$ is concave.

Proof. The "if" part appears in [3] (in fact, for $a=0$ and $b=\infty$, but it also works for any $a<b$ ). More precisely, it was proved there that $\Psi_{1}$ is convex. But this means that assertion (ii') from Proposition 1 is satisfied, and so also is (i).

We now show the converse implication. First assume that $\varphi \in C_{2}(U) \cap \mathcal{C}^{2}$. In this case we follow the idea of [3, Lemma 3]. Consider $F: U \times U \rightarrow \mathbb{R}$ defined by

$$
F(x, y)=\frac{\varphi(x)+\varphi(y)}{2}-\varphi\left(\frac{x+y}{2}\right) .
$$

If a random variable $Z:\{-1,1\} \rightarrow U$ attains two values $x$ and $y$ then $\Psi_{1}(Z)=F(x, y)$. Therefore Proposition $1\left((\mathrm{i}) \Rightarrow\left(\mathrm{ii}^{\prime}\right)\right)$ implies that $F$ is convex. Since $F$ is $\mathcal{C}^{2}, D^{2} F$ is non-negative definite. Thus

$$
\frac{\partial^{2} F}{\partial x^{2}}(x, y)=\frac{1}{2} \varphi^{\prime \prime}(x)-\frac{1}{4} \varphi^{\prime \prime}\left(\frac{x+y}{2}\right) \geq 0 .
$$

Since $\varphi \in C_{2}(U) \subseteq C_{1}(U)$, we have $\varphi^{\prime \prime} \geq 0$ and the above easily implies that if $\varphi^{\prime \prime}\left(x_{0}\right)=0$ for some $x_{0} \in U$, then also $\varphi^{\prime \prime}(x)=0$ for $x \in$ $\left(\left(a+x_{0}\right) / 2,\left(b+x_{0}\right) / 2\right)$. Applying this argument inductively we get $\varphi^{\prime \prime} \equiv 0$, i.e. $\varphi$ is affine. So further we assume $\varphi^{\prime \prime}>0$. The non-negativity of $D^{2} F$ implies that

$$
\frac{\partial^{2} F}{\partial x^{2}} \frac{\partial^{2} F}{\partial y^{2}} \geq \frac{\partial^{2} F}{\partial x \partial y}
$$

and one easily checks that this is equivalent to the concavity of $1 / \varphi^{\prime \prime}$ considered at the points $x, y$ and $(x+y) / 2$. 
Now we show that the assumption $\varphi \in C_{2}(U)$ implies that $\varphi \in \mathcal{C}^{2}$. For $\varepsilon>0$ let $U^{\varepsilon}=(a+\varepsilon, b-\varepsilon)$ and define $\varphi_{\varepsilon}: U^{\varepsilon} \rightarrow \mathbb{R}$ as the convolution $\varphi_{\varepsilon}=\varphi * \eta_{\varepsilon}$, where $\eta_{\varepsilon} \geq 0$ is a smooth approximation of $\delta_{0}$ with $\operatorname{supp}\left(\eta_{\varepsilon}\right) \subseteq$ $(-\varepsilon, \varepsilon)$. Since $C_{2}(U)$ is a convex cone, $\varphi_{\varepsilon} \in C_{2}\left(U^{\varepsilon}\right)$.

Since $\varphi_{\varepsilon}$ is smooth, the first part of the proof implies that $\varphi_{\varepsilon}$ is either affine, or has a strictly positive second derivative with $1 / \varphi_{\varepsilon}^{\prime \prime}$ concave. Then it is easy to see that $\varphi_{\varepsilon}^{\prime \prime}$ is a convex function. Indeed, the affine case is obvious, and if $\varphi_{\varepsilon}^{\prime \prime}>0$ then the concavity of $1 / \varphi_{\varepsilon}^{\prime \prime}$ considered at the points $x, y$ and $(x+y) / 2$ gives

$$
\varphi_{\varepsilon}^{\prime \prime}\left(\frac{x+y}{2}\right) \leq \frac{2 \varphi_{\varepsilon}^{\prime \prime}(x) \varphi_{\varepsilon}^{\prime \prime}(y)}{\varphi_{\varepsilon}^{\prime \prime}(x)+\varphi_{\varepsilon}^{\prime \prime}(y)} \leq \frac{\varphi_{\varepsilon}^{\prime \prime}(x)+\varphi_{\varepsilon}^{\prime \prime}(y)}{2} .
$$

Therefore $\varphi_{\varepsilon}^{\prime \prime} \geq 0$ and for some $x_{0} \in \mathbb{R}, \varphi_{\varepsilon}^{\prime \prime}$ is non-increasing on $\left(-\infty, x_{0}\right] \cap U$ and non-decreasing on $\left[x_{0}, \infty\right) \cap U$, so $\varphi_{\varepsilon}^{\prime}$ is a non-decreasing, concave-convex function.

First we show that $\varphi \in \mathcal{C}^{1}$. Since $\varphi \in C_{2}(U) \subseteq C_{1}(U), \varphi$ is convex, so it is well-known that $\varphi$ has a first derivative on a set $\mathcal{D}_{\varphi}$ with $\mathcal{N} \mathcal{D}_{\varphi}=U \backslash \mathcal{D}_{\varphi}$ countable (so $\mathcal{N} \mathcal{D}_{\varphi}$ is of zero Lebesgue measure and $\mathcal{D}_{\varphi}$ is dense in $U$ ). Moreover, $\varphi^{\prime}$ is continuous at all points of $\mathcal{D}_{\varphi}$ and $\varphi$ is locally Lipschitz. Therefore Lebesgue's dominated convergence theorem yields

$$
\begin{aligned}
\varphi_{\varepsilon}^{\prime}(x) & =\lim _{h \rightarrow 0} \int \frac{\varphi(x-y+h)-\varphi(x-y)}{h} \eta_{\varepsilon}(y) d y \\
& =\left(\varphi^{\prime} * \eta_{\varepsilon}\right)(x) \quad \text { for } x \in U^{\varepsilon}
\end{aligned}
$$

( $\varphi^{\prime}$ is defined a.e.). Taking $\varepsilon \rightarrow 0$, by continuity of $\varphi^{\prime}$ in $\mathcal{D}_{\varphi}$,

$$
\lim _{\varepsilon \rightarrow 0} \varphi_{\varepsilon}^{\prime}(x)=\varphi^{\prime}(x) \quad \text { for } x \in \mathcal{D}_{\varphi} \text {. }
$$

Now fix any decreasing sequence $\varepsilon_{k} \rightarrow 0(k=0,1, \ldots)$ and think of $\varepsilon_{0}$ as small. Below we consider the $\varphi_{\varepsilon_{k}}$ defined on one domain $U^{\varepsilon_{0}}$. The functions $\varphi_{\varepsilon_{k}}^{\prime}$ are non-decreasing and concave-convex and they pointwise converge on the dense set $U^{\varepsilon_{0}} \cap \mathcal{D}_{\varphi}$. This implies that they are also uniformly equicontinuous on any compact interval $\left[a_{0}, b_{0}\right] \subset U^{\varepsilon_{0}}$. Indeed, taking any $a_{i}, b_{i} \in U^{\varepsilon_{0}} \cap \mathcal{D}_{\varphi}(i=1,2)$ such that $a_{1}<a_{2} \leq a_{0}$ and $b_{0} \leq b_{1}<b_{2}$, we see that for sufficiently large $k$ the Lipschitz constant of $\varphi_{\varepsilon_{k}}^{\prime}$ is less than

$$
\max \left(\frac{\varphi^{\prime}\left(a_{2}\right)-\varphi^{\prime}\left(a_{1}\right)+1}{a_{2}-a_{1}}, \frac{\varphi^{\prime}\left(b_{2}\right)-\varphi^{\prime}\left(b_{1}\right)+1}{b_{2}-b_{1}}\right) .
$$

Therefore the Arzelà-Ascoli theorem implies that there exists a subsequence $\varepsilon_{k_{l}}$ such that $\varphi_{\varepsilon_{k_{l}}}^{\prime}$ converges uniformly on $\left[a_{0}, b_{0}\right]$ to some continuous function, which has to be the derivative of $\varphi$. Letting $\varepsilon_{0} \rightarrow 0$ and $a_{0} \rightarrow a, b_{0} \rightarrow b$ we get $\varphi \in \mathcal{C}^{1}$. Moreover, $\varphi^{\prime}$ is also a non-decreasing, concave-convex function.

The proof that $\varphi \in \mathcal{C}^{2}$ is similar. The equality (8) gives $\varphi_{\varepsilon}^{\prime \prime}=\left(\varphi^{\prime} * \eta_{\varepsilon}\right)^{\prime}$ and (9) applied for $\varphi^{\prime}$ instead of $\varphi$ (this is justified since $\varphi^{\prime}$ is a concave-convex 
function and all the facts concerning the derivative of $\varphi^{\prime}$ and the set $\mathcal{D}_{\varphi^{\prime}}$ hold true as in the case of a convex function) yields

$$
\varphi_{\varepsilon}^{\prime \prime}(x)=\left(\varphi^{\prime} * \eta_{\varepsilon}\right)^{\prime}(x) \rightarrow \varphi^{\prime \prime}(x) \quad \text { for } x \in \mathcal{D}_{\varphi^{\prime}} .
$$

Now using the fact that $\varphi_{\varepsilon}^{\prime \prime}$ is convex, a similar argument shows that the convex functions $\varphi_{\varepsilon_{k}}^{\prime \prime}$ are uniformly equicontinuous on compact intervals. As a consequence, some subsequence $\varphi_{\varepsilon_{k_{l}}}^{\prime \prime}$ is uniformly convergent on compact intervals to some continuous function, which has to be the derivative of $\varphi^{\prime}$.

TheOrem 2. Let $U \subseteq \mathbb{R}^{d}$ be an open, convex set. Then for all $n \geq 3$,

$$
C_{n}(U)=\left\{\varphi: U \rightarrow \mathbb{R} \mid \varphi(x)=Q(x)+v^{*}(x)+c\right\},
$$

where $Q$ is a non-negative definite quadratic form on $\mathbb{R}^{d}, v$ is a linear functional on $\mathbb{R}^{d}$ and $c \in \mathbb{R}$.

Proof. The inclusion $\supseteq$ is easy. Since the expectation commutes with $v^{*}$, we can assume $\varphi(x)=Q(x)$. Moreover, we can take $U=\mathbb{R}^{d}$, because if $\varphi \in C_{n}(U)$ and $U^{\prime} \subseteq U$ then $\varphi_{\mid U^{\prime}} \in C_{n}\left(U^{\prime}\right)$.

We show that if $\varphi(x)=Q(x)$ is a quadratic form then

$$
\Psi_{n}(Z)=\Psi_{n}\left(Z-E_{n} Z\right) .
$$

Indeed, denote by $Q(x, y)$ the bilinear form associated with $Q(x)$; then $(2)$ yields

$$
\begin{aligned}
& \Psi_{n}\left(Z-E_{n} Z\right)=E_{n} \Psi_{n-1}\left(Z-E_{n} Z\right)-\Psi_{n-1}(0) \\
& =E_{n} \sum_{K \subseteq\{1, \ldots, n-1\}}(-1)^{|K|} E_{K^{\mathrm{c}}} Q\left(E_{K}\left(Z-E_{n} Z\right)\right) \\
& =\sum_{K \subseteq\{1, \ldots, n-1\}}(-1)^{|K|} E_{K^{\mathrm{c}}} E_{n}\left(Q\left(E_{K} Z\right)-2 Q\left(E_{K} Z, E_{K \cup\{n\}} Z\right)+Q\left(E_{K \cup\{n\}} Z\right)\right) \\
& =\sum_{K \subseteq\{1, \ldots, n-1\}}(-1)^{|K|} E_{K^{\mathrm{c}}}\left(E_{n} Q\left(E_{K} Z\right)-2 Q\left(E_{n} E_{K} Z, E_{K \cup\{n\}} Z\right)\right. \\
& \left.\left.=\sum_{K \subseteq\{1, \ldots, n-1\}}(-1)^{|K|}\left(E_{K^{\mathrm{c}} \cup\{n\}} Q\left(E_{K} Z\right)-Q\left(E_{K \cup\{n\}} Z\right)\right)=\Psi_{n}(Z) .\{n\} Z\right)\right)
\end{aligned}
$$

Now, by induction on $n$, we prove that $\Psi_{n} \geq 0$, i.e. $Q \in C_{n}\left(\mathbb{R}^{d}\right)$. Obviously, $\Psi_{1} \geq 0$. Then the formulas (10) and (2) imply that

$$
\Psi_{n}(Z)=\Psi_{n}\left(Z-E_{n} Z\right)=E_{n} \Psi_{n-1}\left(Z-E_{n} Z\right)-\Psi_{n-1}(0) \geq 0,
$$

since by the induction hypothesis $\Psi_{n-1}\left(Z-E_{n} Z\right) \geq 0$ a.s.

The inclusion $\subseteq$ is more tricky. First, Proposition 2 allows us to consider the case $n=3$ only. The argument presented below is due to K. Oleszkiewicz and is reproduced here with his kind permission. (The author's argument 
was a bit more complicated and was not so general-it worked e.g. for $U=$ $(0, \infty) \subseteq \mathbb{R}$ but not for finite intervals $)$.

First, assume that $\varphi \in C_{3}(U)$ is $\left(\mathcal{C}^{\infty}\right)$ smooth. We define $X:\{-1,1\}^{3}$ $\rightarrow \mathbb{R}$ by

$$
X\left(\varepsilon_{1}, \varepsilon_{2}, \varepsilon_{3}\right)= \begin{cases}3 & \text { if }\left|\varepsilon_{1}+\varepsilon_{2}+\varepsilon_{3}\right|=3, \\ -1 & \text { otherwise. }\end{cases}
$$

Fix $a \in U$ and $v \in \mathbb{R}^{d}$. For $\varepsilon \in \mathbb{R}$, we define $Z_{\varepsilon}=a+v \varepsilon X$. If $|\varepsilon|$ is sufficiently small, $Z_{\varepsilon}$ has values in $U$. The hypothesis implies that $\Psi_{3}\left(Z_{\varepsilon}\right) \geq 0$. On the other hand, if we put $f(x)=\varphi(a+v x)$ for $x$ from some open interval containing 0 , we obtain

$$
\begin{aligned}
\Psi_{3}\left(Z_{\varepsilon}\right) & =\sum_{K \subseteq\{1,2,3\}}(-1)^{|K|} E_{K^{\mathrm{c}}} f\left(\varepsilon E_{K} X\right) \\
& =\frac{1}{4} f(3 \varepsilon)-\frac{3}{2} f(\varepsilon)+2 f(0)-\frac{3}{4} f(-\varepsilon) .
\end{aligned}
$$

Notice that the right hand side vanishes if we take $1, x$ or $x^{2}$ as $f(x)$, and is equal to 6 for $f(x)=x^{3}$. Since $f$ is smooth, applying Taylor's expansion $f(x)=f(0)+f^{\prime}(0) x+\frac{1}{2} f^{\prime \prime}(0) x^{2}+\frac{1}{6} f^{\prime \prime \prime}(0) x^{3}+o\left(x^{3}\right)$ to (11) we obtain

$$
\lim _{\varepsilon \rightarrow 0} \frac{\Psi_{3}\left(Z_{\varepsilon}\right)}{\varepsilon^{3}}=f^{\prime \prime \prime}(0) .
$$

Since $\Psi_{3}\left(Z_{\varepsilon}\right) / \varepsilon^{3} \geq 0$ for $\varepsilon>0$ and $\Psi_{3}\left(Z_{\varepsilon}\right) / \varepsilon^{3} \leq 0$ for $\varepsilon<0$, we obtain $f^{\prime \prime \prime}(0)=0$, hence $D_{v, v, v}^{3} \varphi(a)=0$ for any $v \in \mathbb{R}^{\bar{d}}$ and $a \in U$, so $D^{3} \varphi \equiv 0$. An elementary reasoning shows that $\varphi$ is of the desired form-we leave the details to the reader. (A similar result dealing with functions on an infinitedimensional vector space was given e.g. in [5]. That result says that if a function restricted to any line is a one-variable polynomial of degree at most $k$, then the whole function is a polynomial of degree at most $k$.)

The general case (without assuming $\varphi$ to be smooth) follows easily from the above. For $\varepsilon>0$, we define

$$
U^{\varepsilon}=\{x \in U: \bar{B}(x, \varepsilon) \subseteq U\} .
$$

Clearly, $U^{\varepsilon}$ is an open, convex subset of $U$. Define $\varphi_{\varepsilon}: U^{\varepsilon} \rightarrow \mathbb{R}$ as the convolution $\varphi_{\varepsilon}=\varphi * \eta_{\varepsilon}$, where $\eta_{\varepsilon} \geq 0$ is a smooth approximation of $\delta_{0}$ with $\operatorname{supp}\left(\eta_{\varepsilon}\right) \subseteq B(0, \varepsilon)$. Since $C_{3}(U)$ is a convex cone, $\varphi_{\varepsilon} \in C_{3}\left(U^{\varepsilon}\right)$ and so $\varphi_{\varepsilon}$ is a "quadratic function". Passing to the limit we conclude that so also is $\varphi$.

The following proposition states what the "tensorization property" for the classes $C_{n}(U)$ means.

Proposition 3. Let $\varphi \in C_{n+1}(U)(n \geq 1)$. Let $\mu_{k}^{0}$ and $\mu_{k}^{1}$ for $k=$ $1, \ldots, n$ be probability measures. Then for any $Z: \bigotimes_{k=1}^{n}\left(\mu_{k}^{0} \otimes \mu_{k}^{1}\right) \rightarrow U$ such 
that $E|Z|<\infty$ and $E|\varphi(Z)|<\infty$ we have

$$
\Psi_{n}(Z) \leq E \sum_{A \subseteq\{1, \ldots, n\}} \Psi_{n}^{A}(Z)
$$

where $\Psi_{n}^{A}(Z)$ means the functional $\Psi_{n}$ applied to $Z$ considered as a random variable defined on the product $\bigotimes_{k=1}^{n} \mu_{k}^{I_{A}(k)}$ with all coordinates $\omega_{k}^{1-I_{A}(k)}$ fixed.

Proof. We shall prove that for $Z:\left(\mu_{1}^{0} \otimes \mu_{1}^{1}\right) \otimes \mu_{2} \otimes \cdots \otimes \mu_{n} \rightarrow U$ (satisfying appropriate integrability conditions) one has

$$
\Psi_{n}(Z) \leq E\left(\Psi_{n}^{0}(Z)+\Psi_{n}^{1}(Z)\right)
$$

where $\Psi_{n}^{0}(Z)$ means $\Psi_{n}$ applied to $Z$ considered as a random variable defined on the product $\mu_{1}^{0} \otimes \mu_{2} \otimes \cdots \otimes \mu_{n}$ with $\omega_{1}^{1}$ fixed (and similarly for $\Psi_{n}^{1}(Z)$ ). Labelling the product coordinates $\omega_{1}^{0}, \omega_{1}^{1}, \omega_{2}, \ldots, \omega_{n}$ as $1^{0}, 1^{1}, 2, \ldots, n$ respectively we have

$$
\begin{aligned}
\Psi_{n}(Z) & =\sum_{\substack{K \subset\left\{1^{0}, 1^{1}, 2, \ldots, n\right\} \\
\left|K \cap\left\{1^{0}, 1^{1}\right\}\right| \neq 1}}(-1)^{|K|} E_{K^{\mathrm{c}}} \varphi\left(E_{K} Z\right), \\
E \Psi_{n}^{0}(Z) & =\sum_{K \subset\left\{1^{0}, 2, \ldots, n\right\}}(-1)^{|K|} E_{\left\{1^{1}\right\} \cup K^{\mathrm{c}}} \varphi\left(E_{K} Z\right), \\
E \Psi_{n}^{1}(Z) & =\sum_{K \subset\left\{1^{1}, 2, \ldots, n\right\}}(-1)^{|K|} E_{\left\{1^{0}\right\} \cup K^{\mathrm{c}}} \varphi\left(E_{K} Z\right),
\end{aligned}
$$

and we easily check that $E \Psi_{n}^{0}(Z)+E \Psi_{n}^{1}(Z)-\Psi_{n}(Z)=\Psi_{n+1}(Z)$.

Now observe that it suffices to apply the above argument recursively.

Acknowledgments. I would like to thank Prof. Krzysztof Oleszkiewicz for introducing me to the subject and all the inspiring suggestions including his elegant arguments reproduced in the proof of Theorem 2. I am also indebted to Prof. Stanisław Kwapień for several comments which allowed me to improve the text of this paper.

\section{References}

[1] W. Beckner, A generalized Poincaré inequality for Gaussian measures, Proc. Amer. Math. Soc. 105 (1989), 397-400.

[2] S. Boucheron, O. Bousquet, G. Lugosi and P. Massart, Moment inequalities for functions of independent random variables, Ann. Probab. 33 (2005), 514-560.

[3] R. Latała and K. Oleszkiewicz, Between Sobolev and Poincaré, in: Geometric Aspects of Functional Analysis, Lecture Notes in Math. 1745, Springer, Berlin, 2000, 147-168.

[4] M. Ledoux, On Talagrand's deviation inequalities for product measures, ESAIM Probab. Statist. 1 (1997), 63-87. 
[5] S. Mazur und W. Orlicz, Grundlegende Eigenschaften der polynomischen Operationen. Erste Mitteilung, Studia Math. 5 (1934), 50-68.

[6] K. Oleszkiewicz, Generalization of the hypercontraction method and the KhinchinKahane inequalities, Master's Thesis, Warsaw Univ., 1994 (in Polish).

Paweł Wolff

Institute of Mathematics

Warsaw University

Banacha 2

02-097 Warszawa, Poland

E-mail: pwolff@mimuw.edu.pl

Received January 26, 2007;

received in final form June 14, 2007 